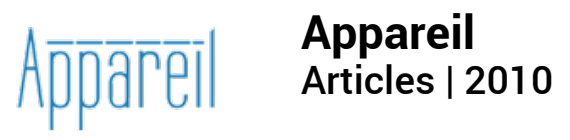

\title{
À propos du texte de Hans Finsler
}

Le développement de la vision photographique

Michel Porchet

\section{OpenEdition}

\section{Journals}

Édition électronique

URL : http://journals.openedition.org/appareil/956

DOI : 10.4000/appareil.956

ISSN : 2101-0714

Éditeur

MSH Paris Nord

Référence électronique

Michel Porchet, « À propos du texte de Hans Finsler », Appareil [En ligne], Articles, mis en ligne le 11 février 2010, consulté le 30 juillet 2020. URL : http://journals.openedition.org/appareil/956 ; DOI :

https://doi.org/10.4000/appareil.956

Ce document a été généré automatiquement le 30 juillet 2020.

\section{(c) (i) ()}

Appareil est mis à disposition selon les termes de la Licence Creative Commons Attribution - Pas d'Utilisation Commerciale - Pas de Modification 4.0 International. 


\title{
À propos du texte de Hans Finsler
}

\author{
Le développement de la vision photographique
}

\section{Michel Porchet}

1 Cet article se situe dans un travail de réflexion sur l'image dans la page imprimée, réflexion conduite alors que s'achève la période de remise en cause de la chaîne graphique commencée il y a une cinquantaine d'année avec l'intervention de la photocomposition et la fin du « marbre ».

2 Dans le cadre du Mois de la photo 2006, précisément consacré à la relation entre la photographie et l'image imprimée, j'ai, avec Irène Attinger, présenté une exposition sur la revue $\boldsymbol{D u}$, un des lieux privilégié du rapport entre l'image photographique ou picturale et la page imprimée, un des hauts lieux du photo-journalisme des années 1950 -1960 .

3 Ce mensuel culturel de grand format et de très haute qualité d'impression a été fondé, en mars 1941, par l'éditeur et imprimeur zurichois Conzett \& Huber, comme vitrine de qualité technique, mais aussi comme vecteur d'affirmation nationale. Cette date peut étonner, mais le paradoxe n'est qu'apparent. La guerre, qui écarte la redoutable concurrence des arts graphiques allemands, incontestables leaders mondiaux, offre des opportunités sur le plan commercial. De plus, en Suisse, l'heure est à l'affirmation nationale. En 1941, les suisses allemands étaient les derniers germanophones qui n'avaient pas été «anschlussés » au grand Reich ou occupés. Dans le cas de Du, cette affirmation nationale se veut celle d'une Suisse ouverte sur le monde. Ceci se traduit par le choix, comme premier rédacteur en chef, d'un homme de gauche : Arnold Kübler qui avait eu l'honneur de voir son journal, le Zürcher Illustrierte interdit par la régime nazi dès 1932.

4 Duest resté la revue d'une certaine élite culturelle. La grande liberté de ton de la revue et son ouverture aux tendances contemporaines ne doivent pas faire illusion. L'abonnement à la revue était un objet de prestige s'adressant à une élite sociale bénéficiant de revenus confortables, un coup d'œil aux publicités qu'elle contient suffit pour s'en convaincre. Certains prétendent que la liberté de la revue était due au fait que ses abonnés ${ }^{1}$ ne la lisaient pas mais l'exposaient plus ou moins ostensiblement dans leur salon puis leur bibliothèque. 
Les élèves de Hans Finsler, professeur de photographie à l'école des arts appliqués de Zurich, en particulier Emil Schulthess et Werner Bischof sont à l'origine de la question posée, en 1953, par le magazine US Camera : "Are Swiss photographers the world's best?». Arnold Kübler, qui reprochait à l'école de Finsler d'être loin du monde, envoya ses élèves en reportage. Cette fusion de la manipulation parfaite des moyens techniques propre à la Nouvelle Photographie et de thématiques liées à l'humain est une spécificité de la photographie suisse de l'immédiat après-guerre. Les photographes de Du, Werner Bischof en particulier, vivaient alors pour une bonne part des travaux que leur commandaient des organisations humanitaires ${ }^{2}$. Dans $\mathbf{D u}$, le photojournalisme se place dans une temporalité particulière, celle de la construction/ reconstruction. Le reportage de Bischof sur l'Europe de l'est s'étale sur plusieurs mois, les photographies sont publiées deux ans après la prise de vue. Lors de la construction d'un barrage en haute montagne, la rédaction fait mettre en place une plate-forme qui sera utilisée plus de deux ans plus tard pour prendre une photo du barrage achevé.

\section{La « qualité suisse » : une routine névrotique}

Rapidement devenues routinière, la qualité technique de cette photographie rejoint la "qualité suisse ", un leitmotiv conservateur. Cette "qualité » était véritablement un comportement névrotique des Suisses de l'époque. La lutte contre l'idéologie aliénante du travail bien fait et du " propre en ordre » sera au cœur des critiques dans les années 1960. Seuls Bischof et Burri, très engagés au côté des luttes de libération nationale, échapperont à la critique radicale conduite durant ces années là. Il n'en ira pas de même pour Schulthess et ses photographies impeccablement composées ou pour Tuggener réputé conservateur sur le plan politique. Malgré ce mouvement de critique, les photographes suisses mettront près de cinquante ans pour s'émanciper de l'influence des artistes de cette période. La "redécouverte ", très tendance, de certains photographes de l'époque, se fonde sur un large oubli de la critique des années 1960. Je suis un peu effaré par la fascination qu'exercent les images de Schulthess, par exemple, sur les professionnels de la photographie ou de l'art à qui je les ai montrées. Les remises en cause des années 1960 prennent désormais un parfum passéiste. 

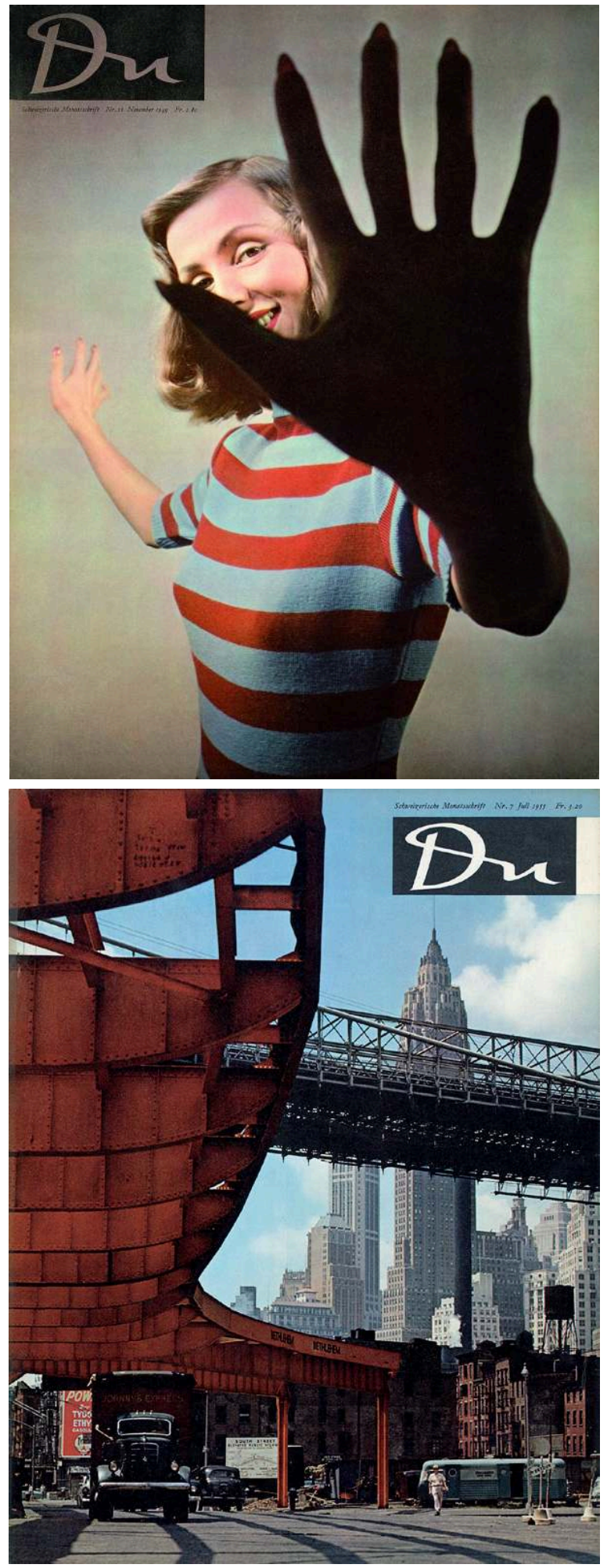

7 Emil Schulthess :

8 Éclat et misère de la perspective (1949) ${ }^{3}$; Voie express F. D. Roosevelt, NY (1955)

9 J'avancerai l'hypothèse que si Robert Frank, comme il l'a souvent dit, se sentait à l'étroit en Suisse, ce n'était pas de l'espace social ou géographique qu'il s'agissait (des Bischof et des Burri en étaient fort loin) mais bien de l'espace esthétique dominé par ce 
que l'on peut décrire comme le « dipôle Kubler-Finsler ». Frank a quitté la Suisse pour échapper à cet espace esthétique et il y est parvenu dès Les Américains ${ }^{4}$.

\section{Hans Finsler : photographe, enseignant et théoricien}

Les noms de certains des élèves de Finsler comme Werner Bischof, René Burri et indirectement Robert Frank, suffisent à situer l'importance de l'enseignant. Né en 1891 et mort en 1972, Hans Finsler a fait des études d'architecture puis d'histoire de l'art à Stuttgart et Munich. Parmi ses professeurs, il faut citer le spécialiste de l'art baroque Heinrich Wölfflin. Il est ensuite bibliothécaire et chargé de cours d'histoire de l'art à l'école d'arts appliqués de Halle. Regrettant l'absence de documentation pour les travaux d'élèves, il développe en autodidacte, dès 1926, un style fonctionnel de photographie documentaire et établit une classe de photographie. Sa première exposition, Neue Wege der Photographie (1928), fut très remarquée. En tant que pionnier de la "Nouvelle Photographie ", il participe aux côtés de László Moholy-Nagy et d'Albert Renger-Patzsch à l'exposition Film und Foto du Werkbund allemand, qui fut également présentée à Zurich en 1929. En 1932, il crée la première classe de photographie à l'école des arts appliqués de Zurich. Il prendra sa retraite en 1958.

11 Finsler ne présenta que très rarement ses propres travaux. Il ne reste de son travail que quelques tirages des années 1920-1930, ses négatifs ayant été détruits. Pour lui l'œuf ${ }^{5}$, l'œuf de l'anthropologue Johan-Jakob Bachofen, est l'objet idéal de la photographie. Il remet en cause l'effet de réel de la photographie et recherche les lois formelles qui conduisent à l'objet photographique.

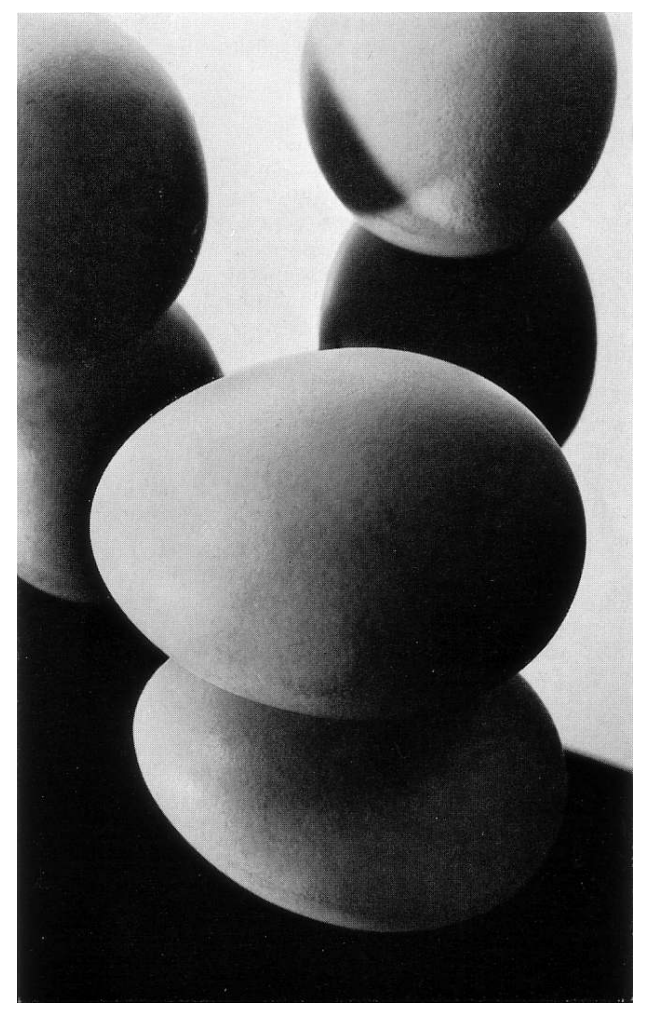

12 Hans Finsler : Æufs 
Dans l'essai, Ma voie pour la photographie ${ }^{6}$, Finsler pose, par l'image, quatre questions :

1. Qu'est-ce que la photographie?

Fondements et aboutissements de la technique photographique.

2. Quelles sont les lois des objets que je photographie?

Lois des choses qui peuvent être rendues visibles en se combinant aux lois de la photographie.

3. La photographie évalue-t-elle les choses? Mettre en évidence les objets secondaires et par moment visibles.

4. La photographie perçoit-elle différemment de l'œil humain?

L'orientation par le regard est toujours liée à l'ici et maintenant, c'est différent pour la photographie et ouvre à la manipulation.

\section{Hans Finsler : l'image de la photographie}

Ce texte de Hans Finsler est paru dans Du en $1964^{7}$. Ce n'est pas un texte théorique, mais le reflet d'un enseignement. Le texte est, en plus, destiné à un public cultivé mais pas spécialisé. Il illustre par trois tableaux et par des portraits photographiques l'évolution de la sensibilité, de la perception de l'espace et du temps en fonction de l'époque. 

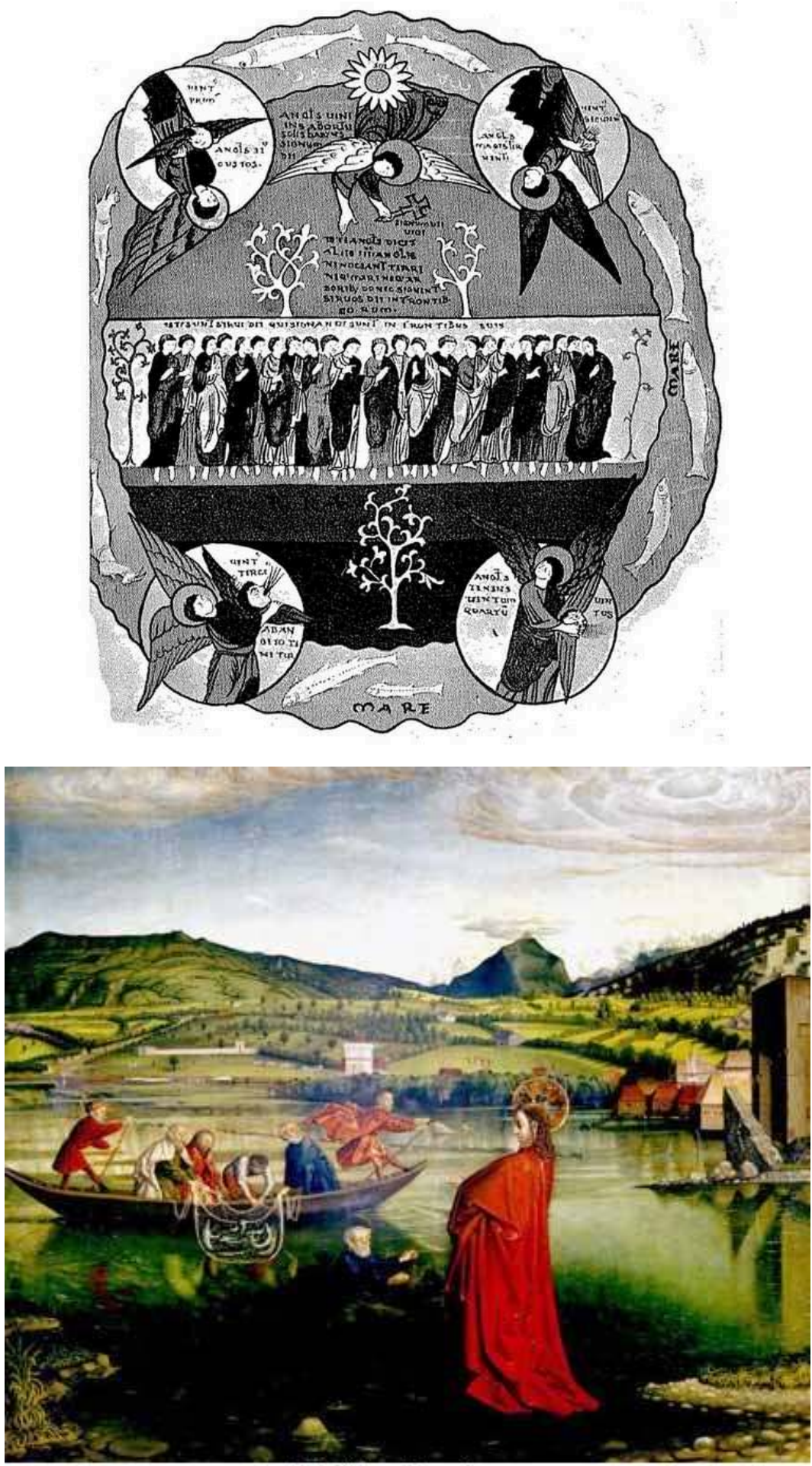

La Pêche miraculeuse

(6) Musées d'art et d'histoire de la Ville de Genève

15 Le Beatus ou Apocalypse de Saint-Sever (milieu XI ${ }^{\mathrm{e}}$ siècle) ; Konrad Witz :La pêche miraculeuse (1444) 


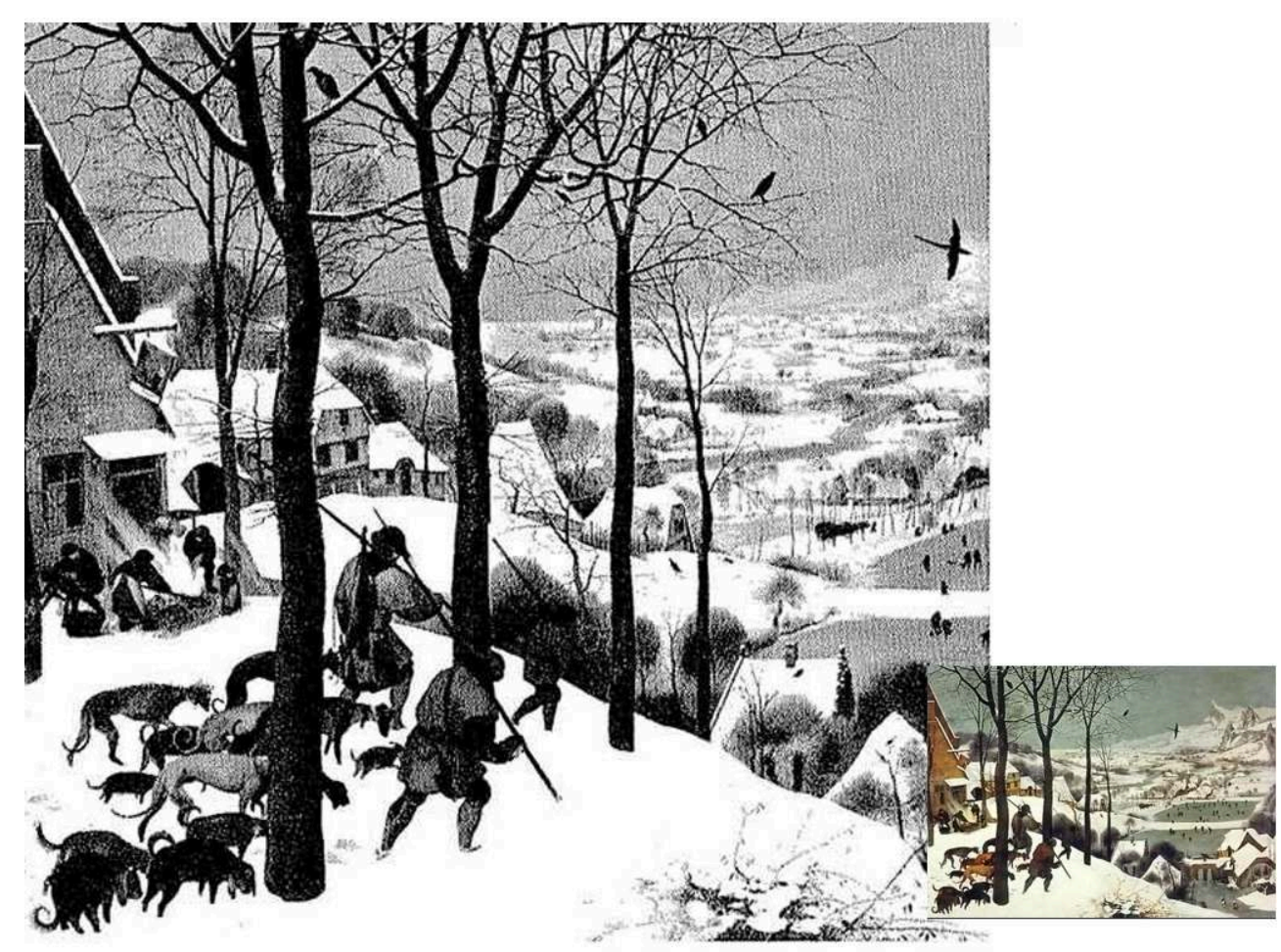

16 Pierre Brueghel : Les chasseurs dans la neige (1565)

17 Les légendes de Finsler soulignent que :

«Dans le Beatus ou Apocalypse de Saint-Sever, datant du milieu du XI siècle, il n'y a ni hasard, ni perspective d'un point de vue personnel, le ciel et la terre forment un cercle et ce qui se passe dans l'espace et le temps universel est dès le début déterminé.» Le tableau de Konrad Witz «montre un espace déterminés, directement intelligible, mais comme au Moyen-âge, le temps traverse l'image dans des événements traités comme des paraboles. Si nous savons où quelque chose se produit, nous ignorons quand." Sur le tableau Renaissance de Pierre Bruegel : «Point de vue et temps personnels sont définis, bien que ce lieu et ce temps n'existent pas. Ce n'est pas la réalité mais un événement typique dans un espace typique, expression de beaucoup d'événements dans beaucoup d'espaces. »

Il montre aussi, à partir de portraits photographiques, que si les techniques influencent l'image, les transformations ne suivent pas directement les évolutions techniques.

«Au début, les prises de vue montrent les hommes isolés dans le temps et l'espace. L'espace est créé avec les accessoires de décors du théâtre de la solitude. L'homme est avec ses vêtements du dimanche, hors des vicissitudes de la vie quotidienne, il domine ses émotions, il a une ferme conscience de lui-même, il est l'image formée d'une époque. Montrer les changements internes des événements par l'expression d'un visage ne fait, au début, pas partie des tâches d'une photographie. »

19 On peut attribuer cela à l'influence de la peinture et/ou aux limites techniques de la photographie des débuts (les temps de pose nécessairement longs). Il n'en reste pas moins que l'évolution du portrait photographique est liée à une évolution culturelle et elle ne se fait que bien après qu'elle soit devenue techniquement possible. 


\section{La main, donc la conscience, forme l'image}

« La main crée la forme comme symbole de notre existence. Rien n'est formé qui ne repose sur une conscience. L'image de la peinture naît de la reconnaissance d'une relation entre des signes et le dasein. "

" On hésite à appliquer le mot image à la photographie. On parle d'illustration, c'est à dire de reproduction, ou de prise de vue, on prend ce qui est déjà là. La main manque à la photographie. Nous ne pouvons pas comprendre le monde sans avoir un concept, nous ne pouvons le saisir, le fixer - les mots sont aussi liés à la main -, sans que la main crée des signes, la langue crée des sons et les sons doivent à nouveau devenir des signes, de l'écrit. »

«La photographie est sans concept: les hommes, la forêt, les étoiles sont là, sans concept, tels qu'ils sont. Ce ne sont pas des images des hommes, de la forêt, des étoiles ce sont eux-mêmes. Sont-ils eux-mêmes? Nous savons qu'ils ne sont pas cela. Nous savons que la photographie fixe à la place de la matière un pigment, à la place du corps une surface, à celle de l'espace un cadre, à celle du temps, de la vie, une durée. Ces différences suffisent-elles à faire la photographie une image ou à devenir une image?»

20 S'il pose la question du rapport à la main à l'image et de l'interrogation que pose la photographie de ce point de vue sa réponse est claire: «Deux conditions sont déterminante pour les nouvelles images : la relation technique conditionnelle de l'appareil à la surface réfléchissante de la chose et la décision subjective de l'homme face à la conscience de l'espace temps qui apparaît dans l'acte de la prise de vue." Il met en évidence les six propriétés de la photographie qui ne correspondent pas à notre vision :

1. La photographie isole un fragment à partir de l'espace et du temps,

2. l'angle de vision,

3. le net et le flou,

4. le mouvement,

5. la couleur,

6. les contrastes.

Ces six propriétés peuvent devenir des moyens de création de l'image photographique.

\section{Les apports réciproques de la peinture et de la photographie}

Les fondements de l'image photographique sont déjà créés au $\mathrm{XVI}^{\mathrm{e}}$ siècle par l'émergence de la projection centrale et l'irruption du " point du sujet ». Il manque encore la couche capturant la lumière, couche dont Finsler souligne qu'elle est un matériau graphique. Finsler montre deux dispositifs projectifs : celui de Dürer et une camera oscura avec la description de Rainer Gemma-Frisius d'une éclipse du soleil de 1544 . 


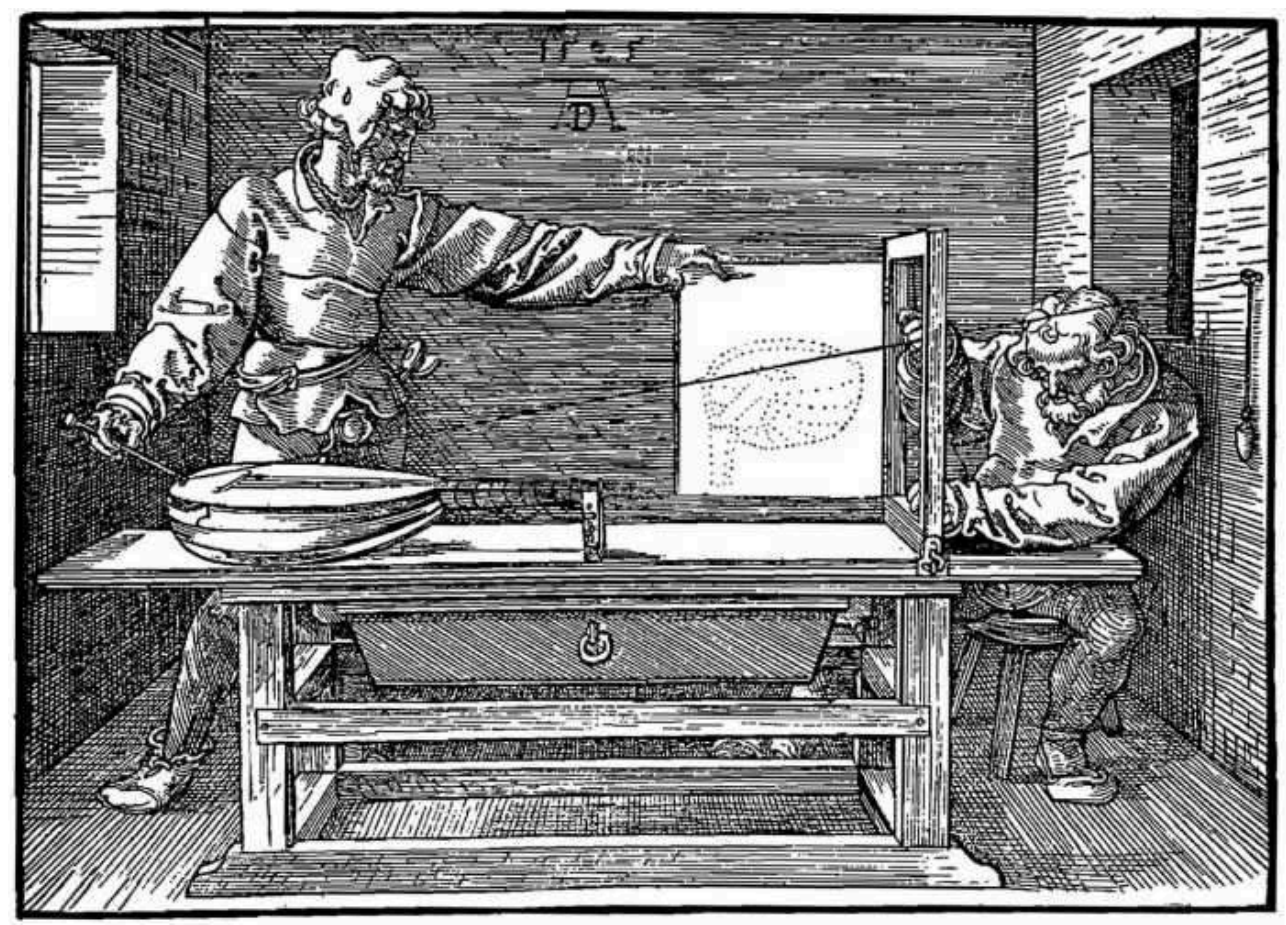

23 Dürer : Le dessin du Luth (1525)

«La photographie a ses propres lois. Les hommes de la gravure sur bois de Dürer Le dessin du Luth de 1525 connaissent ces lois. Ils sont comme dans une prise de vue photographique du même point de vue ou du même point de l'œil, la photographie a seulement automatisé la reproduction. L'image du luth par Dürer n'est pas le luth. C'est un luth dessiné. L'homme le voit ainsi, lorsque il l'observe à partir du point sur la paroi, d'où partent les fils. Il s'établit une nouvelle réalité, la réalité de la perspective, de la photographie, la réalité d'un homme; d'un individu, la réalité subjective de la Renaissance. »

Wölfflin a été le premier historien d'art à avoir utilisé dans ses conférences la projection simultanée de deux œuvres afin de permettre une confrontation directe. Finsler lui emprunte cette méthode d'exposition fondée sur l'opposition de deux images. Il oppose l'entrepont (1907) d'Alfred Stieglitz représentant conséquent de la Straight Photography aux Demoiselles d'Avignon de Picasso, un premier pas vers le cubisme. Stieglitz montre au-delà des personnages, de leur classe sociale marquée par le pont qui leur est assigné, la structure plastique créé par la cheminée, l'escalier, la passerelle, le chapeau de paille rond, qui ouvre une circulation que nul n'emprunte. Pour lui, il y a là " une image de formes qui redonne le sentiment que j'ai de la vie. ». Pour Finsler, l'image de Stieglitz signifie la séparation finale de la peinture et de la photographie mais elle montre en même temps l'exigence commune d'une relation réglée, d'une durabilité. 

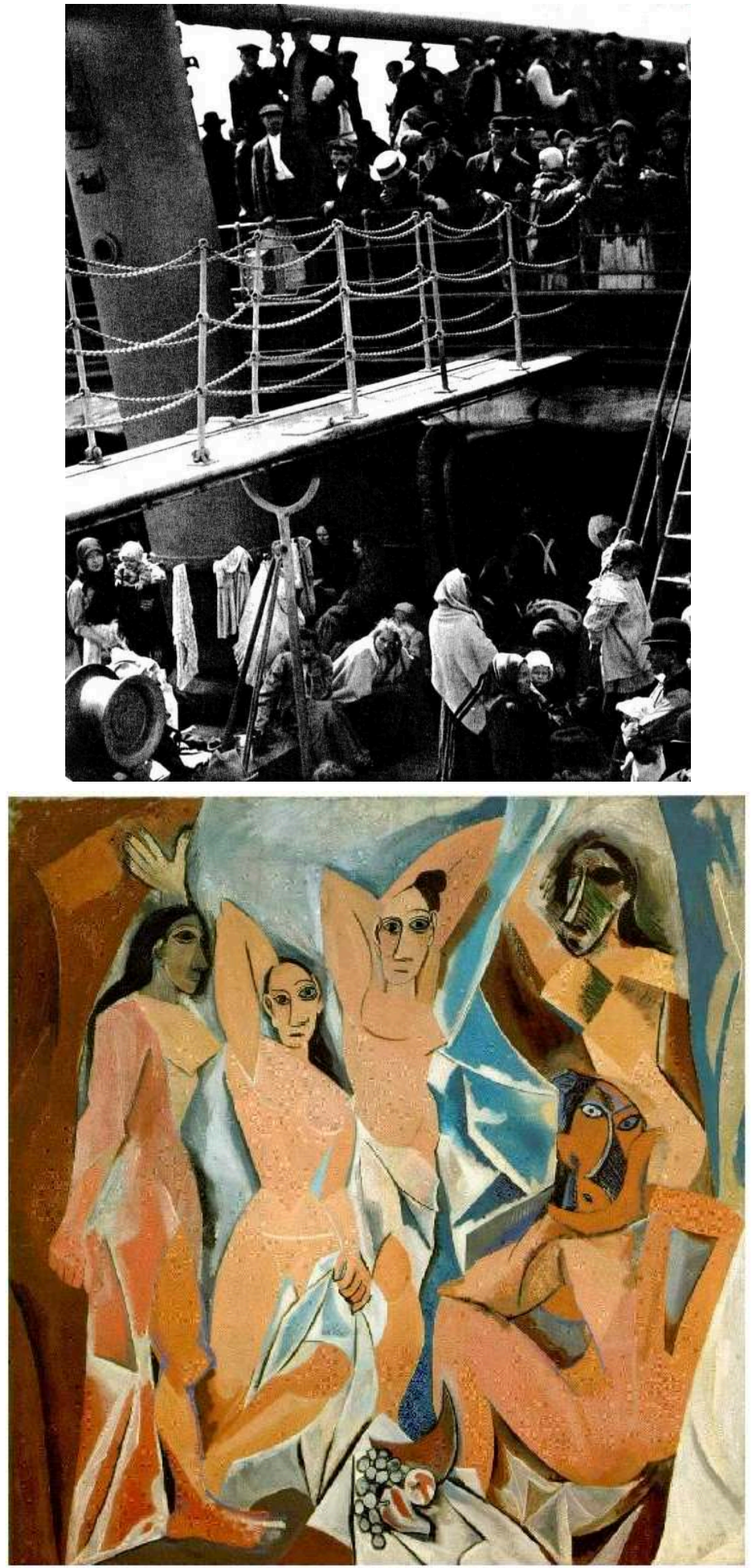

25 Alfred Stieglitz : L'entrepont (1907); Picasso : Les Demoiselles d'Avignon (1907)

26 A propos du cadre, Finsler oppose le Nana de Manet à un autoportrait de Steichen. 


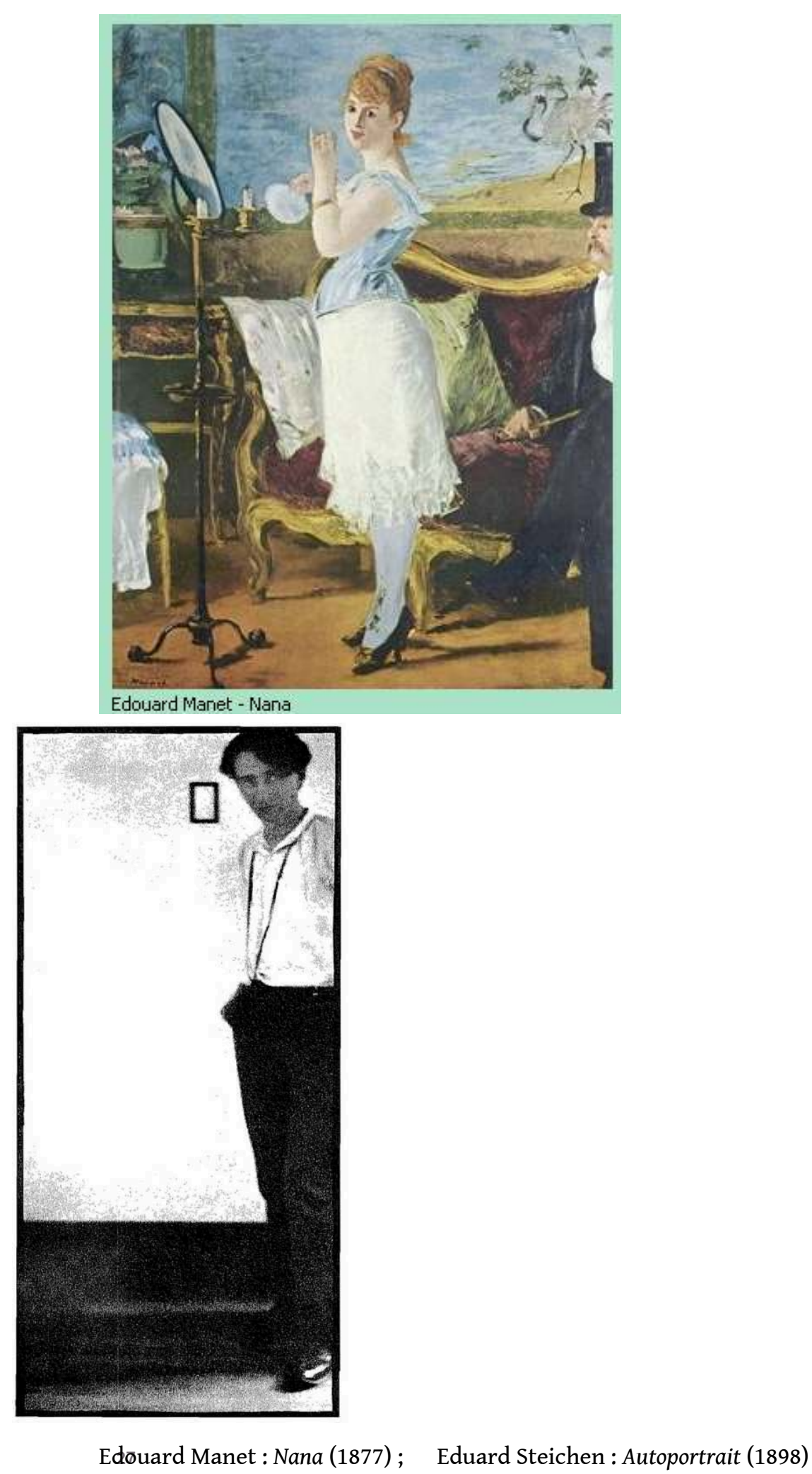

Dans les légendes des images, il précise :

«Le scandale créé par le Nana n'a pas en dernier lieu son origine dans la présence tombée hors du cadre du cavalier. Le cavalier coupé de Nana est une idée de l'image, non une coupure de l'espace. »

«Le cadrage en peinture est différent du cadrage en photographie. L'autoportrait de Steichen, avec le rectangle noir du cadre qui est découpé par le rectangle clair de 
la paroi et celui sombre du sol, avec l'image près de la tête, qui n'est qu'un cadre qui répète le cadre extérieur, ce contraste de forme avec le mouvement de l'homme est préparé, la réalité est posée. Steichen s'opposant à nous, avec ses dix neuf ans, esquisse notre siècle. »

\section{L'instant photographique}

«Dans chaque appareil photographique existent des dispositifs pour pouvoir isoler un fragment d'espace temps. La photographie est liée au concept de temps à la durée et à l'instant. La mise en forme n'est pas seulement création de la forme, c'est également une mise en forme du temps et de l'espace. Dans l'interprétation du temps, le rapport de l'instant à la durée ou au momentané est toujours éphémère pour une durée perceptible. Un changement de notre conception de l'être est lié à un changement de notre conception du temps.»

"Quel temps est celui de la photographie? C'est en premier lieu une négation du temps. La visibilité de l'événement est liée au temps. Il n'y a pas de pause, pas d'interruption dans le cours du temps. L'instant est passage et non suspension. La photographie est suspension. Elle interrompt le cours de la vie. Le soleil ne tourne plus. C'est une condition technique de la photographie, que durant le moment de la prise de vue le temps s'arrête, que rien ne bouge, vu de la captation, le futur est engagé. L'instant photographique est une préparation permanente, une coupe dans le temps. Mais cette interruption du cours du temps, cet instant photographique spécifique, issu du vécu diffère du moment créé par le peintre et nous laisse reconnaître quelque chose du caractère du représenté que sinon nous n'aurions pas reconnu. Le photographe voit différemment du peintre. L'événement accouche de lui-même. Nous pouvons montrer la réalité au travers de la réalité. Le factum se dresse près de la fiction. »

Finsler évoque, par l'intermédiaire de Bischof, les questionnements du photojournalisme Le Bischof de 1947, celui de l'enfant hongrois croit à l'action positive du photojournalisme, le sérieux, la tranquillité attristée, la lumière forment le visage, l'espace est neutre et il n'y a aucun chevauchement. Un tel optimisme paraît justifié quand, en 1951, après la publication par Life Magazin d'un reportage qu'il a fait en Inde, le Congrès américain vote des mesures immédiates d'aide. Le Bischof de 1952, traumatisé par ses reportages sur la guerre de Corée, devient sceptique par rapport à son propre travail. L'étudiant à Kyoto révèle cette inquiétude sceptique : l'expression du visage, la cigarette, mais surtout le fort chevauchement qu'entraîne l'escalier et la silhouette fantomatique qui s'éloigne dans le fond parachèvent une dimension spectrale de l'image. Si l'étudiant de Bischof peut nous rapprocher du monde de Robert Frank, c'est une autre voie qu'empruntera Bischof à la fin de sa vie, celle d'un retour aux pratiques de sa jeunesse, proches de la « Nouvelle Objectivité ». 

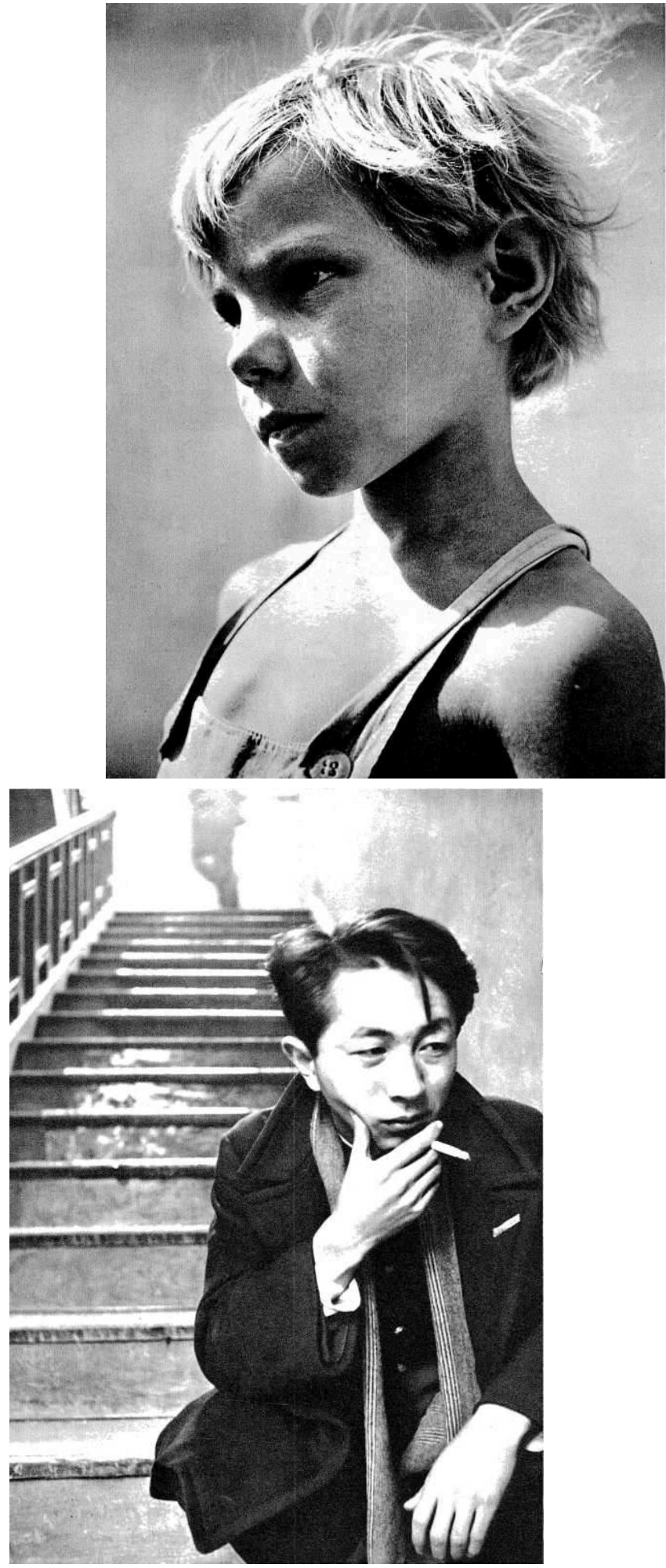

Werner Bischof : L'enfant hongrois (1947) ; Étudiant à Kyoto (1952)

\section{En conclusion :}

31 Le tsunami du 26 décembre 2004 peut être considéré comme l'événement fondateur d'une transformation des pratiques des industries de l'information au travers d'un 
abandon du photojournalisme au profit d'un recours systématisé aux vues prises par M. Toulemonde dans l'édition et la communication. Le texte de Finsler a retenu mon attention dans un tel moment parce qu'il souligne l'intervention d'une conscience dans la confection d'une image et expose la relation de la photographie à la réalité et à la subjectivité. Le passage suivant est le point central du texte de Finsler :

« Nous ne pouvons comparer la photographie avec la réalité, nous devons à la place de la réalité à nouveau prendre la photographie. Nous sommes à peine conscient du point auquel, malgré leurs liens, photographie et réalité sont différentes. Une reproduction fidèle de la réalité, la reproduction photographique exacte d'un dessin par exemple, ne dit rien de la réalité. Ce n'est qu'à travers la dissemblance de la photographie et de la réalité malgré leur sujétion mutuelle que la photographie peut être une image de la réalité, peut prendre position sur la réalité. »

Évidemment cela n'épuise pas le sujet, mais « dissemblance » et "sujétion mutuelle » forment un bon couple dialectique. Les points importants selon moi sont les suivants :

- la question, de première importance, du rapport fondateur de l'image à la main et de l'interrogation que pose la photographie de ce point de vue,

34 - la mise en évidence de l'historicité de la perspective, de l'apport de la peinture à la photographie (en soulignant que les peintres $\mathrm{du} \mathrm{XvI}{ }^{\mathrm{e}}$ siècle inventent la photographie), mais aussi de la relation des deux à la sensibilité de l'époque (le rapprochement Stieglitz Picasso par exemple).

Finsler pose, à propos de la photographie, des bonnes questions (la relation de l'image au monde et à l'espace temps, l'instant photographique et la temporalité de la photographie, le cadre, le champ et le hors champ, ...). Les réponses qu'il donne dans le texte sont souvent limitées, mais l'image apporte un message qui lui est propre. Son travail est un exemple de la raison graphique, d'un exposé rationnel qui ne recourt pas exclusivement au discours. Le texte formule des interrogations et les réponses sont à rechercher dans les images. Par cette méthode il peut aborder des questions essentielles concernant la photographie, mais aussi l'image en général. Par exemple, il met très bien en évidence l'opposition entre l'esthétique de Bischof et celle de Frank :

«Dans le travail de Bischof, la conscience d'une norme est toujours présente, même quand elle se montre d'abord dans la déformation. Le reflet anamorphosé des formes rigides du gratte-ciel dans la laque de la voiture agit comme un symbole d'une partie de notre vie actuelle. Robert Frank montre les faits de l'existence, sans les juger, des faits avec lesquels nous avons à nous occuper. Les prises de vue sont non résolues, non libérées d'un monde plein de questions, qui nous sont posées ». 

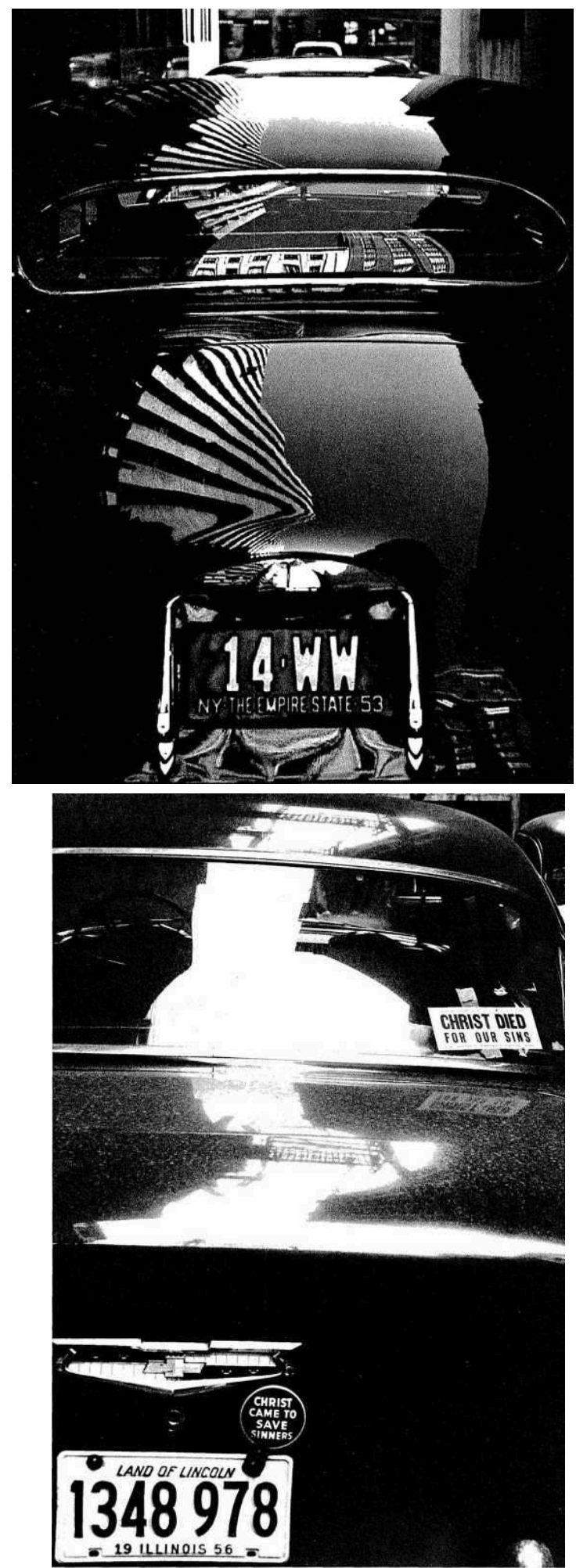

36 Werner Bischof : Gratte-ciel dans la laque d'une automobile; Robert Frank : Chicago (1956) 


\section{NOTES}

1. La diffusion atteignait une trentaine de milliers d'exemplaires aux meilleurs moments

2. Ce n'est pas une nouveauté, il y a eu auparavant l'enquête organisée aux Etats-Unis par la Farm Security Administration avec certains des plus grands noms : Dorothea Lange, Walker Evans, Carl Mydans, Gordon Parks, Jack Delano... Les relations entre le grand photojournalisme et l'action humanitaire de longue durée mériteraient une étude fine.

3. Image reprise en couverture du Figaro SCOPE de la semaine du 25 au 31 octobre 2006

4. Robert Frank : Les Américains. Ed. Robert Delpire, Paris 1958.

5. L'œuf, comme le marbre ou la peau humaine, remet en cause la notion de surface de l'optique vulgaire, en effet, la réflexion se fait au-delà de la surface de l'objet.

6. Hans Finsler : mein Weg zur Fotografie / My Way to Photography. Pendo-verlag, Zurich 1971

7. Du, mars 1964, numéro thématique intitulé : Hans Finsler:Das Bild der Photographie (Hans Finsler : L'image de la photographie). Le titre de l'article de Finsler est Die Entwicklung des Photographischen Sehens (Le développement de la vision photographique). Toutes les citations de Finsler sont tirées de cet article et traduites de l'allemand par l'auteur du présent texte.

\section{RÉSUMÉS}

Les noms de certains des élèves de Finsler comme Werner Bischof, René Burri et indirectement Robert Frank, suffisent à situer l'importance de l'enseignant. Hans Finsler, élève du spécialiste de l'art baroque Heinrich Wölfflin, développe en autodidacte, dès 1926, un style fonctionnel de photographie documentaire et établit une classe de photographie. En tant que pionnier de la «Nouvelle Photographie », il participe aux côtés de László Moholy-Nagy et d'Albert RengerPatzsch à l'exposition Film und Foto du Werkbund allemand. En 1932, il crée la première classe de photographie à l'école des arts appliqués de Zurich. Il prendra sa retraite en 1958. Finsler ne présenta que très rarement ses propres travaux. Il ne reste de son travail que quelques tirages des années 1920 - 1930, ses négatifs ayant été détruits. Remettant en cause l'effet de réel de la photographie, il recherche les lois formelles qui conduisent à l'objet photographique. Dans ses textes, il pose les questions de fond concernant la photographie et ses objets comme celle du rapport fondateur de l'image à la main et de l'interrogation que pose la photographie de ce point de vue. Il y répond, généralement par l'image.

\section{INDEX}

Mots-clés : durée, Fragment, image, instant, Nouvelle Photographie, page imprimée, peinture, perception, photographie, projection centrale, sensibilité, signes, techniques 